\title{
The basis of the Ponzano-Regge-Turaev-Viro-Ooguri quantum-gravity model is the Loop Representation basis
}

\author{
Carlo Rovelli \\ Physics Dept. University of Pittsburgh, Pittsburgh PA 15260, USA \\ and Dipart. di Fisica Universita' di Trento and INFN sez. Padova, Italia \\ rovelli@pittvms.bitnet
}

July 25, 2018

\begin{abstract}
We show that the Hilbert space basis that defines the Ponzano- ReggeTuraev-Viro- Ooguri combinatorial definition of 3-d Quantum Gravity is the same as the one that defines the Loop Representation. We show how to compute lengths in Witten's 3-d gravity and how to reconstruct the 2-d geometry from a state of Witten's theory. We show that the non-degenerate geometries are contained in the Witten's Hilbert space. We sketch an extension of the combinatorial construction to the physical 4-d case, by defining a modification of Regge calculus in which areas, rather than lengths, are taken as independent variables. We provide an expression for the scalar product in the Loop representation in 4-d. We discuss the general form of a nonperturbative quantum theory of gravity, and argue that it should be given by a generalization of Atiyah's topological quantum field theories axioms.
\end{abstract}


The problem of describing physics at the Planck scale and the quantum properties of gravity, is the problem of understanding what is a non- trivial generally covariant quantum field theory. The last years have seen many developments in our understanding of these theories: Witten's introduction of topological field theories, in their two versions, a' la Chern- Simon [1] and a' la Donaldson [2]; Atiyah's axiomatization of these [3]; dynamical triangulations techniques [4]; Ashtekar reformulation of general relativity [5], which opened the way to the Loop Representation [6], which lead to discover solutions of the Wheeler-DeWitt equation, the relevance of Knot Theory in quantum gravity and a discrete structure of space at the Planck scale [7]; Turaev and Viro's [8] reformulation of the Ponzano-Regge model [9] in terms of quantum groups, which provides a combinatorial definition of 3- d topological field theories; Crane and Vetter's [10] extension of this construction to 4-d; the pioneering work of Ooguri, which in 3-d has tied the Euclidean, combinatorial, canonical and topological definitions of quantum gravity [11], and in 4-d has opened the path to the Crane-Vetter work. These results share a remarkable common flavor, besides, of course, the common long term aim of quantizing gravity. In this paper, we find the bridge between the 3 - $d$ Ponzano-Regge-Turaev-Viro-Ooguri (PRTVO) model and the Loop Representation, we discuss the physical interpretation of 3-d quantum gravity, and we sketch a general theory of physical 4-d gravity in which all these lines may converge.

Ponzano and Regge [9] considered Regge-calculus [13] in 3-d, but made the ansatz that the lengths $l_{i}$ of the Regge-calculus links be constrained to be half integers: $l_{i}=j_{i}=1 / 2 n_{i}$ (integer $n_{i}$ ). Half integers $j_{i}$ can be interpreted as labels of $\mathrm{SU}(2)$ representations. The Regge calculus action can then be written as a very simple expression, which is essentially a sum over the tetrahedra of the triangulation of the 6 -j symbols of the 6 (half- integer) lengths $l_{i}=j_{i}$ of the links of each tetrahedron. The partition function of Quantum Gravity can then be constructed by fixing a sufficiently thin triangulation $\Delta$, and summing over its colorings c (assignments of half integers to every link). The reason for taking half-integer lengths, as well as the relation between lengths of links and $\mathrm{SU}(2)$ representations appeared to be quite mysterious at the time. In this paper we throw some light on this relation. Turaev and Viro [8] were able to show that the Ponzano-Regge partition function is independent from the triangulation chosen (and transformed it in a finite sum by replacing $\mathrm{SU}(2)$, with a corresponding quantum group with 
a finite number of representations). Ooguri [11] has related the quantization of 3-d quantum gravity based on this model to the Witten quantization of the same theory. Ooguri construction can be summarized in short as follows. The quantum states of the Ponzano-Regge theory have to be taken, following Atiyah's general formulations of topological quantum field theories, as quantum combinations $\Phi_{\Delta}(c)$ of the colored triangulations $(\Delta, c)$ induced on the 2-d boundary $\partial M$ of the 3-d manifold $M$. In Witten theory, quantum states are wave functions $\Psi(\omega)$ over the moduli space of the flat $\mathrm{SU}(2)$ connections $A_{a}^{I}(x)$ on the 2-d boundary ( $\omega$ being equivalent classes of $A_{a}^{I}(x)$ 's). Ooguri relates the two representations of the theory by

$$
\Psi(\omega)=\sum_{c} \Phi_{\Delta}(c) \Psi_{\Delta, c}(\omega)
$$

where we have absorbed in $\Phi_{\Delta}(c)$ a normalization factor appearing in eq.(16) of Ref. [11]. The "matrix elements of the change of basis"

$$
\langle\omega \mid \Delta, c\rangle=\Psi_{\Delta, c}(\omega)
$$

will described in a moment. Louis Crane and Lee Smolin have suggested that there may be a direct relation between the Ooguri construction and the Loop Representation [14]; in this letter, we show, indeed, that (1) is nothing but the Loop Transform [6], which relates the connection representation of Quantum Gravity with the Loop Representation. The Ooguri representation $\Phi_{\Delta}(c)$ is therefore essentially equivalent to the Loop Representation. In the making this relation explicit, we will provide a physical interpretation of the Ponzano-Regge ansatz according to which the Regge-calculus links have halfinteger length and are related to $\mathrm{SU}(2)$ representations, and we will find the physical justification of the Ooguri construction.

The functions $\Psi_{\Delta, c}(\omega)$ introduced by Ooguri, are constructed as follows. Given the triangulation $\Delta$, we construct the trivalent graph dual to $\Delta$. The $\operatorname{arc} C_{i}$ of this graph crosses the i-th link of the triangulation $\Delta$; we associate to $C_{i}$ the $\mathrm{SU}(2)$ representation $j_{i}$, where $j_{i}$ is the half-integer that colors the i-th link of $\Delta$. We then associate to $C_{i}$ the function over flat $\mathrm{SU}(2)$ connections $A_{a}^{I}$ given by the Wilson line $U_{j_{i}}\left[A, C_{i}\right]=\mathcal{P} \exp \left\{\int_{C_{i}} A_{a}^{I} t_{j_{i}}^{I} d x^{a}\right\}$, where the $\mathrm{su}(2)$ generators $t_{j_{i}}^{I}$ are taken in the $j_{i}$ representation. Next, we consider the product of all these Wilson lines, where 6-j symbols are used to contract the indices at the trivalent intersections. The resulting object is a 
function of the triangulation $\Delta$, the coloring $c$ and the (flat) connection $A$. It is gauge invariant, and thus it defines a function over the moduli space of the flat $\mathrm{SU}(2)$ connections for every $(\Delta, c)$; this function is $\Psi_{\Delta, c}(\omega)$.

In order to relate this construction with the Loop Representation, the first observation is that, since any representation of $\mathrm{SU}(2)$ is obtained by tensor multiplication of the $j=1 / 2$ representation with itself, a Wilson line $U_{j}\left[A, C_{i}\right]$ in the $\mathrm{j}$ representation can be expressed by means of $2 j$ Wilson lines $U_{1 / 2}\left[A, C_{i}\right]$ in the $1 / 2$ representation. We exploit this fact by replacing each $\operatorname{arc} C_{i}$ of the trivalent graph with precisely $2 j_{i}$ lines, each carrying a $U_{1 / 2}\left[A, C_{i}\right]$ parallel transport matrix. Accordingly, the sum at the trivalent intersections obtained with the 6-j symbols, can simply be replaced by the sum over all the possible rootings of these lines at the intersection. Finally, $\Psi_{\Delta, c}(\omega)$ can be reexpressed as a combination of products of traces of holonomies of A along the resulting closed loops, all taken in the $1 / 2$ representation. This follows from elementary properties of $\mathrm{SU}(2)$ representation theory. In other words, the colored triangulation $(\Delta, c)$ uniquely determines an ensemble $E_{\Delta, c}=\left\{\alpha_{1}, \alpha_{2}, \ldots\right\}$ of multiple loops (sets of closed loops) $\alpha_{i}=\left(\alpha_{i 1}, \alpha_{i 2}, \ldots \alpha_{i N}\right)$, where $\alpha_{i j}$ are (single) loops; each multiple loop $\alpha_{i}$ having the property that precisely $2 j$ single loops cross a link of the triangulation with color $j$. The ensemble $E_{\Delta, c}$ is defined as the set of all the homotopically inequivalent multiple loops with this property. By construction we have the main relation:

$$
\Psi_{\Delta, c}(\omega)=\sum_{\alpha_{i} \in E_{\Delta, c}} \prod_{i} \operatorname{Tr} U_{1 / 2}\left[A, \alpha_{i j}\right]
$$

Now, given a multiple loop $\alpha_{i}$, the product $\prod_{i} \operatorname{Tr} U_{1 / 2}\left[A, \alpha_{i j}\right]$ is nothing but the loop state $\left|\alpha_{i}\right\rangle$, written in the connection representation, namely

$$
\left\langle A \mid \alpha_{i}\right\rangle=\Psi_{\alpha_{i}}=\prod_{i} \operatorname{Tr} U_{1 / 2}\left[A, \alpha_{i j}\right]
$$

This relation is at the roots of the Loop Representation. Using this relation, and its gauge invariance, we have

$$
\langle\omega \mid \Delta, c\rangle=\Psi_{\Delta, c}(\omega)=\sum_{\alpha_{i} \in E_{\Delta, c}}\left\langle A \mid \alpha_{i}\right\rangle=\sum_{\alpha_{i} \in E_{\Delta, c}}\left\langle\omega \mid \alpha_{i}\right\rangle,
$$

or

$$
|\Delta, c\rangle=\sum_{\alpha_{i} \in E_{\Delta, c}}\left|\alpha_{i}\right\rangle
$$


Eq. (3) provides an identification between the Loop representation basis states $|\alpha\rangle$ and the Ooguri states $|\Delta, c\rangle$. The relation is many-to-one because the loop states are not independent (they form an overcomplete basis). This relation is our first result.

In Ooguri's work, the relation (1) is postulated, and the equivalence of the combinatorial theory with Witten's quantization is derived a posteriori by showing the isomorphism of the two structures. Still, the half-integer lengths remain as mysterious as they were in the original Ponzano-Regge paper. To provide an interpretation of this fact, let us calculate the lengths of the links of a triangulation in a fixed quantum state of the gravitational field. A recent calculation in $(3+1)$-d gravity, shows that the area of any surface is quantized in the Loop Representation in multiples of $1 / 2$ (in Planck units); the area being precisely given by the number of intersections of the surface with the loops of the quantum state. It is natural to suspect that a similar relation may work in one dimension less. In fact, let us show it does. The length $l$ of a curve $C$ in 3 -d gravity is given by

$$
l[C]=\int_{C} d t \sqrt{\frac{d C^{a}}{d t} \frac{d C^{b}}{d t} g_{a b}}=\int_{C} d t \sqrt{\frac{d C^{a}}{d t} \frac{d C^{b}}{d t} E^{I c} E^{I c} \epsilon_{a c} \epsilon_{b d}},
$$

where $E^{I a}$ is the variable conjugate to the connection and $\epsilon_{a c}$ is the antisymmetric two dimensional pseudotensor. We refer to [6] for the notation. In order to promote $l[C]$ to an operator, we have to deal with the product of the two E's. Following Ref. [7], we point split the product $E E$, by means of the gauge invariant two-point object

$$
\operatorname{Tr}\left[U\left[A, \gamma_{x}^{\prime \epsilon}\right] E^{a}\left(\gamma_{x}^{\epsilon}(0)\right) U\left[A, \gamma_{x}^{\prime \prime \epsilon}\right] E^{b}\left(\gamma_{x}^{\epsilon}(\pi)\right)\right]==T^{a b}\left[\gamma_{x}^{\epsilon}\right](0, \pi)
$$

Here $\gamma_{x}^{\epsilon}$ is a loop with radius $\epsilon$, and center in $x, \gamma_{x}^{\prime \epsilon}$ and $\gamma^{\prime \prime \prime}{ }_{x}$ are its two components from the value 0 to $\pi$ and from $\pi$ to 0 of the loop parameter, and in the second equality we have introduced the standard Loop Representation notation [6] for this operator. Note that classically we have

$$
\lim _{\epsilon \rightarrow 0} T^{a b}\left[\gamma_{x}^{\epsilon}\right](0, \pi)=\operatorname{det} g g^{a b}(x)
$$

The operator corresponding to the observable $T^{a b}\left[\gamma_{x}^{\epsilon}\right](0, \pi)$ is well defined; using the Loop Representation, it is given [6] by

$\langle\alpha| T^{a b}\left[\gamma_{x}^{\epsilon}\right](0, \pi)==\int_{\alpha} d s \frac{d \alpha^{a}}{d s} \delta^{2}\left(\alpha(s), \gamma_{x}^{\epsilon}(0)\right) \int_{\alpha} d u \frac{d \alpha^{b}}{d t} \delta^{2}\left(\alpha(u), \gamma_{x}^{\epsilon}(\pi)\right) \sum_{i}\left\langle\alpha \# \#_{s u, i} \gamma_{x}^{\epsilon}\right|$ 
Following ref. [7], we may regularize the delta functions by a further replacement of $\gamma_{x}^{\epsilon}$ by means of a one parameter family of loops. We can then compute the action of the operator $l[C]$ on a loop state $\langle\alpha|$. The square root of the square of the (regularized) delta function gives an absolute value; in the limit the intersection rearrangement gives just a multiplicative factor, and taking the limit we obtain, in Planck units,

$$
\left\langle\alpha\left|l[C]=\frac{1}{2} \int_{\alpha} d s \int_{C} d t\right| \frac{d \alpha^{a}}{d s} \frac{d C^{b}}{d t} \epsilon_{a b}\right|\langle\alpha|
$$

The double integral is precisely the (positive) intersection number between $C$ and $\alpha$. Thus we arrive at the following results: i. the length of every curve $C$ is quantized in units of $1 / 2$; ii. if the gravitational field is in the state $\langle\alpha|$, the length of a curve $C$ is given (reinserting conventional units) by

$$
l[C]=n[C, \alpha] \frac{L_{\text {Planck }}}{2},
$$

where $n[C, \alpha]$ is the number of times $\alpha$ crosses $C$.

Now we can return to the PRTVO model. Result i. above implies that summing over independent states in quantum gravity means to sum just over quantized half-integer lengths, precisely as in the Ponzano Regge ansatz. Moreover, the relation between the 2-dimensional colored triangulation on the boundaries of the manifold $\mathrm{M}$ and the Witten theory is now physically transparent: Recall that the i-th link $C_{i}$ of the triangulation, with has color $j_{i}$ is crossed precisely by $2 j_{i}$ loops, thus $n\left[C_{i}, \alpha\right]=2 j_{i}$; therefore, using result ii., the physical length in Planck units in the quantum state defined by these loops is

$$
l_{i}=1 / 2 n[C, \alpha]=\frac{1}{2} 2 j_{i}=j_{i}
$$

Thus, the physical length of the i-th link in Ooguri state is precisely equal to its coloring. Therefore, the state $\Psi_{\Delta, c}(w)$, which Ooguri associates to the colored triangulation $(\Delta, c)$ is a quantum state in which the physical length of the links of the triangulation is precisely equal to their coloring, in Planck units. This is our main result.

An important consequence of this result is the fact that it throws light on the confused issue of the existence of an "unbroken phase" and a "broken phase" in quantum gravity. This issue concerns the existence of states corresponding to non-degenerate metrics in a quantization in which fields are 
excitation around the zero metric configuration (as opposed to flat). The considerations above show that as far as $2+1$ gravity is concerned, the problem does not sussist. Indeed, in an generic state $|\alpha\rangle$, the diffeomorphism-invariant functions of the metric are computable, and, in general, they take values corresponding to non-degenerate metric configurations. The core of the subtelty is the diffeomorphism invariance of the theory. The number of independent invariant observables in the theory is finite in the quantum theory as well as in the classical theory. To clarify this point, let us consider a 2-d space manifold with the topology of a torus. The "no-loop" state $|0\rangle$ gives a vanishing metric tensor. However, let us consider the state $|\alpha, \beta\rangle$, where $\alpha$ and $\beta$ are the two independent non contactible loops of the torus (the corresponding state in the Witten representation is easily obtained from Eq. (2)). In this state, the minimal length of any curve wrapping once around the torus is $\frac{1}{2} L_{\text {Planck }}$. Now let us consider the 2 -d space manifolds of the classical theory. Since spacetime if flat, we can always go to a flat euclidean 2-d manifold by means of a gauge transformation (a 3-d diffeomorphism). The physically meanigful information about space is contained in two numbers that characterise a flat torus. Thus, in this theory an arbitrary non-degenerate flat space metric is characterized by these two variables. Say, for definitness, two radii. Now let us return to the quantum theory. In the state $|\alpha, \beta\rangle$, the two variables have a well-defined non-vanishing value. Thus the quantum state $|\alpha, \beta\rangle$ corresponds to the classical non-degenerate configuration in which space is formed by a flat torus in which the two radii are long $\frac{1}{2} L_{\text {Planck }}$ each. Bigger spaces are obtained by wrapping the loops that define the state more times around the torus. The extrinsic curvature of these spacelike surfaces, then, will of course be completely indetermined, due to Heisenberg principle. This example shows that, at least as far as the $2+1$ theory is concerned, the non-degenerate geometries live in the same Hilbert space as the completely degenerate state.

Before getting to the second part of this work, where we discuss the $3+1$ theory, let us note that the relation between the Loop Representation theory and the PRTVO model allows us to write the scalar product of two loop states $|\gamma\rangle$ and $\left|\gamma^{\prime}\right\rangle$ by means of a sum over colorings: we put $\gamma$ and $\gamma^{\prime}$ on the boundaries of a (topologically trivial) three manifold $\mathrm{M}$, we fix a triangulation $\Delta$, and we have

$$
\left\langle\gamma \mid \gamma^{\prime}\right\rangle=\sum_{c\left(\gamma, \gamma^{\prime}\right)} \prod P R(c)
$$


where the sum is over all the triangulations $c\left(\gamma, \gamma^{\prime}\right)$ such that the coloring of the links of the boundaries is determined by the number of times the loops $\gamma$ and $\gamma^{\prime}$ cross the link, and by $\prod P R(c)$ we indicate the Ponzano-Regge product of the coloring.

The above result indicates a direction for constructing the physical 4-d theory. Let us consider the 4-d manifold $\mathrm{M}$, with, say, two boundaries $\partial M_{1}$ and $\partial M_{2}$. We begin by fixing a 4 -d triangulation $\Delta$ of spacetime, which induces $3-d$ triangulations of the two boundaries. In 4-d, they are the areas of surfaces, not the lengths, that are naturally quantized in $1 / 2$ the Planck unit. Thus, it is natural to use as independent variables for Regge calculus the areas of the faces, rather than the lengths of the links. A key observation is that a 4-d simplex has the same number (10) of faces (2-d symplices) and links (1-d simplicies). Therefore we can generically invert the relation between lengths and areas, and express the lengths of the 10 links of each 4-simplex as functions of the areas of the 10 faces. Let $a_{1} \ldots a_{10}$ be the areas of the 10 faces of a fixed 4-simplex $s$. The Regge action of the simplex can be expressed as a function of these areas: $S_{\text {Regge }}(s)=S_{\text {Regge }}\left(a_{1} \ldots a_{10}\right)$. Note that $S_{\text {Regge }}$ must be a function of 10 variables with the full symmetry of the 4-simplex. We suspect that the corresponding quantity $S_{\text {Regge }}\left(a_{1} \ldots a_{10}\right)$, seen as function of half-integer variables, has an interpretation in terms of group representation theory (at least in the large $a_{i}$ limit) analogous to the 6 -j symbols interpretation of its $3-\mathrm{d}$ analog, but we have not found it. I The above construction defines a combinatorial quantum theory in 4-d (for a fixed triangulation). In the absence of boundaries, we have

$$
Z(\Delta)=\sum_{c} Z_{\text {Regge }}(\Delta, c)=\sum_{c \text { of } \Delta} \prod_{s} \exp \left\{S_{\text {Regge }}(c)\right\}
$$

where the sum is over the colorings, the product over the 4- simplices. The states of this theory are given by the induced colorings of the induced triangulation on the 3-d boundary of the 4-d triangulated spacetime. These states are physically determined by the fact that there is a three dimensional triangulation of space such that the (2-d) surfaces of the triangulation have

\footnotetext{
${ }^{1}$ The reason the group $\mathrm{SU}(2)$ is still the relevant group, in spite of the fact we are one dimension above, is in the very roots of Ashtekar's magic construction: the (complexified) Lorentz group splits naturally in two (complexified) $\mathrm{SO}(3)$ groups, its self-dual and antiselfdual parts, and, as shown by Ashtekar, the full theory can be constructed using only one of the two $\mathrm{SO}(3)$ components.
} 
assigned areas; these areas being half-integers in Planck units. These states are therefore in correspondence with the states of the Loop Representation. The correspondence being given by the result of ref.[7], that the physical area of any surface is $1 / 2$ the number of loops that cross the surface. Since the states can be written in terms of loop states, and viceversa, this theory defines a scalar product in loop space by:

$$
\left\langle\gamma \mid \gamma^{\prime}\right\rangle_{\Delta}=\sum_{c\left(\gamma, \gamma^{\prime}\right) \text { of } \Delta} Z_{\text {Regge }}(\Delta, c)
$$

where $\gamma$ is on $\partial M_{1}$ and $\gamma^{\prime}$ is on $\partial M_{2}$, and the sum is over all the colorings of the interior areas, the colorings of the areas on the boundaries being determined as $1 / 2$ the number of times the loops cross the surface. The idea that states of the Loop Representation can be better understood in terms of the area they induce on a 3-d triangulation was proposed by Lee Smolin [15].

We still do not have a well defined diffeomorphism invariant theory, since the above construction depends on the triangulation $\Delta$. 2 We eliminate this dependence by summing over all the triangulations of $\mathrm{M}$. The key point in this construction is that this is possible to sum over the triangulations, because we have a way of naming the quantum states in the boundaries, which is independent from the triangulation of the boundary itself, namely the loop basis. Thus, we define a theory as

$$
Z=\sum_{\Delta} \sum_{c \text { of } \Delta} Z_{\text {Regge }}(\Delta, c)
$$

and the scalar product between two loop states by

$$
\left\langle\gamma \mid \gamma^{\prime}\right\rangle=\sum_{\Delta}\left\langle\gamma \mid \gamma^{\prime}\right\rangle_{\Delta}
$$

(If in Eq. (14) we perform the sum over the colorings first, the theory takes a form strictly related to the dynamical triangulations approaches to quantum gravity [4]; this relation, we believe, deserves to be better explored.) The important point is that scalar product defined in this way is invariant under

\footnotetext{
${ }^{2}$ An optimistic hope would be that the scalar product above does not depend on the triangulation. While we do not hope for so much, still we do not think this be totally impossible, as some considerations below may suggest.
} 
independent diffeomorphisms on each of the two loops, because so is the sum. Therefore it defines a scalar product $\left\langle K \mid K^{\prime}\right\rangle$ between knot states by

$$
\left\langle K(\gamma) \mid K\left(\gamma^{\prime}\right)\right\rangle=\left\langle\gamma \mid \gamma^{\prime}\right\rangle
$$

These equations provide a formal expression for the Hilbert structure of quantum gravity. We expect that Eq. (5) defines a projector on the knot states, which projects on the solutions of the Wheeler DeWitt equation, as it happens in 3-d [11]. This is our main proposal for a 4-d theory.

To clarify the meaning of Eqs. (4) and (5), let us rewrite them in terms of the original continuous Ashtekar's variables $A$ and $E$. We have P

$$
\left\langle\gamma \mid \gamma^{\prime}\right\rangle=\int\left[d^{4} A\right]\left[d^{4} E\right] \exp \left\{-1 / \hbar S_{E}\left[{ }^{4} A,{ }^{4} E\right]\right\} \Psi_{\gamma}(A) \Psi_{\gamma^{\prime}}^{*}(A)
$$

where

$$
\Psi_{\gamma}(A)=\Psi_{\gamma}\left({ }^{3} A\right)=\left\langle{ }^{3} A \mid \gamma\right\rangle=\operatorname{tr} \mathcal{P} \exp \oint_{\gamma} A
$$

and each loop is in one of the two boundaries of spacetime. It is easy to convince oneself that this is the correct formal expression for the scalar product in the Loop Representation, up the problem of definition and finiteness of the functional integration. To our knowledge, this expression was first suggested by Maurizio Martellini [16]. This is the connection representation analog of Hawking expression [17]

$$
\langle\Psi \mid \Phi\rangle=\int\left[d^{4} g\right] \exp \left\{-1 / \hbar S_{E}\left[{ }^{4} g\right]\right\} \Psi^{*}\left({ }^{3} g\right) \Phi\left({ }^{3} g^{\prime}\right)
$$

where the integration is over all the 4 -d metrics ${ }^{4} g$ on $\mathrm{M}$, and ${ }^{3} g$ and ${ }^{3} g^{\prime}$ are the restrictions of ${ }^{4} g$ to the two components of the boundary of M. Of course these functional integrals do not mean anything until a definition is provided; in particular, if we want to compute them in a perturbation

\footnotetext{
${ }^{3}$ Ooguri suggests [12] that in a one may have two conjugate variables in the continuum version of the theory, which correspond respectively to the choice of the triangulation and the choice of the coloring in the combinatorial version of the theory. Note the similarity between the Ashtekar's action $S[A, E]=\int F \wedge E \wedge E$, with the (topological !) BF theory action $S[A, B]=\int F \wedge B$ which seems to underlie the Ooguri-Crane-Vetter invariants $[10,12]$. The relation between the construction proposed here and the (triangulation independent) Ooguri- Crane-Vetter construction deserves to be studied in detail .
} 
expansion around flat space, we encounter the weel known gravitational divergences. The combinatorial expression given above is a proposal for this definition. I In this continuum case, Hawkings does indeed give a formal derivation of the fact that the functional integral defines a projection on the solutions of the Wheeler DeWitt equation. This is an indirect support of our expectation that Eq.(5) projects on the solutions of the hamiltonian constraint.

We conclude with a consideration on the formal structure of 4-d quantum gravity, which is important to understand the above construction. Standard quantum field theories, as QED and QCD, as well as their generalizations like quantum field theories on curved spaces and perturbative string theory, are defined on metric spaces. Witten's introduction of the topological quantum field theories has shown that one can construct quantum field theories defined on a manifold which has only its differential structure, and no fixed metric structure. The theories introduced by Witten and axiomatized by Atiyah have the following peculiar feature: they have a finite number of degrees of freedom, or, equivalently, their quantum mechanical Hilbert spaces are finite dimensional; classically this follows from the fact that the number of fields is equal to the number of gauge transformations. However, not any diff-invariant field theory on a manifold has a finite number of degrees of freedom. Witten's gravity in 3-d is given by the action $S[A, E]=\int F \wedge E$, which has finite number of degrees of freedom. Consider the action $S[A, E]=$ $\int F \wedge E \wedge E$, in $3+1$ dimensions, for a (self dual) $\mathrm{SO}(3,1)$ connection $A$ and a (real) one form $E$ with values in the vector representation of $\mathrm{SO}(3,1)$. This theory has a strong resemblance with its $2+1$ dimensional analog: it is still defined on a differential manifold without any fixed metric structure, and is diffeomorphism invariant. We expect that a consistent quantization of such a theory should be found along lines which are more similar to the quantization of the $\int F \wedge E$, theory than to the quantization of theories on flat space, based on the Wigthman axioms namely on n- points functions and related objects. Still, the theory $\int F \wedge E \wedge E$ has genuine field degrees of freedom: its physical phase space is infinite dimensional, and we expect that its Hilbert state space will also be infinite dimensional. There is a

${ }^{4}$ Of course, each of the above equations can be immediately generalized to non-trivial topologies, Hartle-Hawking states, disconnected universes, and so on, if one is interested in this kind of physics directions. 
popular belief that a theory defined on a differential manifold without metric and diffeomorphism invariant has necessarily a finite number of degrees of freedom ("because thanks to general covariance we can gauge away any local excitation"). This belief is of course wrong. A theory as the one defined by the action $\int F \wedge E \wedge E$ is a theory that shares many features with the topological theories, in particular, no quantity defined "in a specific point" is gauge invariant; but at the same time it has genuinely infinite degrees of freedom. Indeed, this theory is of course nothing but (Ashtekar's form of) standard general relativity.

The fact that "local" quantities like the n-point functions are not appropriate to describe quantum gravity non-perturbatively has been repeatedly noted in the literature. As a consequence, the issue of what are the quantities in terms of which a quantum theory of gravity can be constructed is a much debated issue. The above discussion indicates a way to face the problem: The topological quantum field theories studied by Witten and Atiyah provide a framework in terms of which quantum gravity itself may be framed, in spite of the infinite degrees of freedom. In particular, Atiyah's axiomatization of the topological field theories provides us with a clean way of formulating the problem. Of course, we have to relax the requirement that the theory has a finite number of degrees of freedom. These considerations leads us to propose that the correct general axiomatic scheme for a physical quantum theory of gravity is simply Atiyah's set of axioms [3] up to finite dimensionality of the Hilbert state space. We denote a structure that satisfies all Atiyah's axioms, except the finite dimensionality of the state space, as a generalized topological theory .

The theory we have sketched is an example of such a generalized topological theory. We associate to the connected components $\partial M_{i}$ of the boundary of $\mathrm{M}$ the infinite dimensional state space of the Loop Representation of quantum gravity. Eq.(5), then, provides a map, in Atiyah's sense, between the state spaces constructe on two of these boundary components. Equivalently, it provides the definition of the Hilbert product in the state space.

One could argue that the framework we have described cannot be consistent, because it cannot allow us to recover the "broken phase of gravity" in which we have a nondegenerate background metric: in the proposed framework one has only non-local observables on the boundaries, while in the broken phase a local field in $\mathrm{M}$ has non-vanishing vacuum expectation value. We think that this argument is weak because it disregards the diffeomor- 
phism invariance of the theory: in classical general relativity no experiment can distinguish a Minkowskian spacetime metric from a non-Minkowkian flat metric. The two are physically equivalent, as two gauge-related Maxwell potentials. For the same reason, no experiment could detect the absolute position of, say, a gravitational wave, (while of course the position of an e.m. wave is observable in Maxwell theory). Physical locality in general relativity is only defined as coincidence of some physical variable with some other physical variable, while in non general relativistic physics locality is defined with respect to a fixed metric structure. In classical general relativity, there is no gauge-invariant obervable which is local in the coordinates. Thus, any observation can be described by means of the value of the fields on arbitrary boundaries of spacetime. This is the correct consequence of the fact that "thanks to general covariance we can gauge away any local excitation", and this is the reason for which one can have the ADM "frozen time" formalism. The spacetime manifold of general relativity is, in a sense, a much weaker physical object than the spacetime metric manifold of ordinary theories. All the general relativistic physics can be read from the boundaries of this manifold. At the same time, however, these boundaries still carry an infinite dimensional number of degrees of freedom.

Finally, we must recall that the computation of the evolution of expectation values in physical time (as opposed to coordinate time, which has no diffeomorphism invariant meaning) requires the use a physical clock coupled to the theory (in principle this could also be a component of the gravitational field itself) [18]. In this sense the integration (or the sum) over the $\mathrm{M}$ is physically very analogous to the derivation of the propagator of a relativistic particle by means of an integral over the paths $x^{\mu}(\tau)$, where $\mu=0,1,2,3$; in the particle case too, indeed, the scalar product between two wave functions on Minkowski space can be obtained by integrating over a five dimensional manifold that interpolates two Minkowski spaces (see for instance [19]). Physical evolution, of course, is in $x_{o}$, not in $\tau$, namely in 4 -d, not in 5-d. In addition, we should also recall that the quantization of the physical area is a non-gauge invariant result, unless reinterpreted in some suitably gauge-invariant context [20].

Summarizing, we have have shown the following:

i. The "colored triangulation" basis of the Ponzano-Regge-Turaev-ViroOoguri quantization of 3-d gravity is precisely the Loop Representation basis. ii. In Witten's theory, we can compute the lengths of the arcs of a Regge 
traingulation, (up to diffeomorphisms).

iii. We can interpret the quantization of the length in half integer units in physical terms: the spectrum of the length operator has discrete half-integer eigenvalues.

iv. These lengths are related to the $\mathrm{SU}(2)$ representations because the quantum states that diagonalize the lengths are given (in the connection representation) by Wilson lines that cross the curve $2 \mathrm{j}$ times, or, equivalently, by one Wilson line in the $\mathrm{j}$ representation that crosses the curve.

Motivated by these results, we have sketched a 4-d combinatorial theory, based on a modification of Regge calculus. Many questions remain open as far as this theory is concerned, the most relevant ones being the relation between the Euclidean and the Lorentzian theory, and the convergence properties of the sum (3). Finally, we have proposed that Atiyah's axiomatization of topological field theories can be extended also to theories with infinite degrees of freedom, and that this extension can be takes as the general formal structure of a quantum theory of gravity.

\section{References}

[1] E Witten, Comm Math Phys 121 (1989) 351; Nucl Phys B322 (1989) 622; B330 (1990) 285; B311 (1988/89) 46; Nucl Phys B340 (1990) 281

[2] E Witten, Comm Math Phys 117 (1988) 353

[3] MF Atiyah, The Geometry and Physics of Knots, Accademia Nazionale dei Lincei, Cambridge University Press 1990; Publ Math Inst hautes Etudes Sci Paris 68 (1989) 175

[4] J Ambjorn, B Durhuus, T Jonsson, Mod Phys Lett A6 (1991) 1133; J Ambjorn, Nucl Phys B25A (1992) 8; J Ambjorn, J Jurkiewicz Phys Lett B278 (1992) 42. ME Agishtein, AA Migdal, Mod Phys Lett A6 (1991) 1863. Godfrey, M Gross Phys Rev D43 (1991) R1749; M Gross, Nucl Phys B20 (1991) 724

[5] A Ashtekar, Phys Rev Lett 57 (1986) 2244; Non-perturbative canonical 
gravity, Lecture notes in collaboration with RS Tate, World Scientific 1991

[6] C. Rovelli, L. Smolin, Phys Rev Lett 61 (1988) 1155; Nucl Phys B331 (1990) 80. For a review, see: C Rovelli, Class Quant Grav 8 (1991) 1613. For the 2+1 dimensional theory see: A Ashtekar, V Husain, C Rovelli, J Samuel, L Smolin, Class and Quant Grav 6 (1989) L185

[7] A. Ashtekar, C. Rovelli, L. Smolin, Phys Rev Lett 69 (1992) 237

[8] V Turaev, O Viro, Topology 31 (1992) 865

[9] G Ponzano, T Regge, "Semiclassical limits of Racah Coefficients" in Spectroscopy and Group theoretical methods in Physics. Ed F Bloch, North Holland, Amsterdam 1968

[10] L Crane, D Yetter, "A categorical construction of 4D topological quantum field theories", Kansas State University preprint 1992

[11] H Ooguri, Nucl Phys B382 (1992) 276

[12] H Ooguri, Mod Phys Lett A7 (1992) 2799

[13] T Regge, Nuovo Cimento 19 (1961) 551

[14] L Smolin, personal communication; L Crane, "Categorical Physics", Kansas State prep 1992

[15] L Smolin, "Time, measurement and information loss in quantum cosmology", Syracuse preprint 1993

[16] M Martellini, personal communication

[17] SW Hawking, in General Relativity, an Einstein centenary Survey, eds. SW Hawking, W Israel, Cambridge University Press 1979

[18] C Rovelli, Class and Quant Grav 8 (1991) 297; 8 (1991) 317; Phys Rev D42 (1991) 2638; D43 442 (1991)

[19] PD Mannheim, Phys Lett 166B (1986) 191

[20] C. Rovelli, "A Generally covariant quantum theory and a prediction on quantum measurement of geometry", sumbitted to Nucl Phys (1992). 